\title{
Lepton-number violation and massless nonorthogonal neutrinos
}

\author{
Paul Langacker* and David London \\ Deutsches Elektronen Synchrotron-DESY, Hamburg, Federal Republic of Germany
}

(Received 18 April 1988)

\begin{abstract}
Mixing between light and heavy neutrinos can induce lepton-flavor and total-lepton-number violation in the light sector, even if the light neutrino masses are zero or extremely small, because the states actually produced in weak processes are generally nonorthogonal. We develop the formalism for describing such nonorthogonal neutrinos and explore the phenomenological implications for neutrino "oscillation" experiments, the solar-neutrino problem, constraints from universality and other charged- and neutral-current experiments, $\mu \rightarrow e \gamma$, and $\Delta L= \pm 2$ effects in muon decay and neutrinoless double-beta decay.
\end{abstract}

\section{INTRODUCTION}

In the standard model with massless neutrinos both the individual lepton-flavor numbers $L_{e}, L_{\mu}$, and $L_{\tau}$ and the total lepton number $L=L_{e}+L_{\mu}+L_{\tau}$ are automatically conserved (up to vacuum tunneling effects which are totally negligible under ordinary circumstances ${ }^{1}$ ). That is because in the absence of neutrino mass terms or new interactions there are no transitions between the SU(2) doublets

$$
\left(\begin{array}{l}
v_{e L}^{0} \\
e_{L}^{-}
\end{array}\right), \quad\left(\begin{array}{l}
v_{\mu L}^{0} \\
\mu_{L}^{-}
\end{array}\right), \quad\left[\begin{array}{l}
v_{\tau L}^{0} \\
\tau_{L}^{-}
\end{array}\right) .
$$

Furthermore, SU(2) symmetry requires that the weakeigenstate neutrinos $v_{e L}^{0}, v_{\mu L}^{0}$, and $v_{\tau L}^{0}$ must be orthogonal. Hence, they can be assigned the lepton-flavor numbers $L_{e}=1, L_{\mu}=1$, and $L_{\tau}=1$, respectively, and the $L_{i}$ (and $L$ ) are conserved.

If the standard model is extended to allow Dirac neutrino masses ${ }^{2}$ [i.e., by adding additional "right-handed" SU(2)-singlet Weyl neutrinos], then the weak eigenstates $v_{i L}^{0}$ will be related to the mass-eigenstate neutrinos $v_{j L}$ by a unitary transformation

$$
v_{i L}^{0}=U_{L i j} v_{j L},
$$

where $U_{L}$ is the leptonic analogue of the CabibboKobayashi-Maskawa quark mixing matrix. Since the weak- and mass-eigenstate neutrinos do not coincide, in general, the individual lepton flavors are no longer conserved: one can have phenomena such as neutrino oscillations ${ }^{2}$ or the incoherent production of definite mass eigenstates.

For Majorana neutrino masses (e.g., generated by a Higgs triplet ${ }^{3}$ or by the seesaw mechanism ${ }^{4}$ ) there is again a unitary transformation relating the weak and mass eigenstates, as in (2), so that the individual lepton flavors are not conserved. In addition, there can be processes, such as neutrinoless double-beta decay $\left(\beta \beta_{0 v}\right)$, which violate the total lepton number by $\Delta L= \pm 2$.

In the course of a recent investigation of the limits on the possible mixing between the ordinary fermions and heavy exotic fermions ${ }^{5}$ we have rediscovered an interesting mechanism for inducing both $L_{i}$ and $L$ violation without introducing masses for the ordinary neutrinos (or for which the masses can be much smaller than is usually considered necessary for observable effects) or new interactions. The idea is quite simple. Because of $\mathbf{S U}(2)$ the weak eigenstates $v_{i L}^{0}$ must be orthogonal. However, in the presence of mixing between light and heavy neutrinos, the light components of the $v_{i L}^{0}$ (which are what are relevant to most weak processes) need not be orthogonal, even if the light neutrino masses are zero or very small. This mechanism has been described before. ${ }^{6-9}$ In the present paper we develop the formalism needed to describe nonorthogonal neutrinos in a very general class of models, discuss the phenomenological implications for $L_{i}$ and $L$ violation in such processes as neutrino appearance experiments, the solar-neutrino problem, $\mu \rightarrow \epsilon \gamma$, muon decay, and $\beta \beta_{0 v}$, and also consider the indirect limits from charged-current universality and other weak processes with no direct violation of lepton number.

A simple example will illustrate the mechanism quantitatively. Consider the two-family standard model to which are added SU(2)-singlet Weyl neutrinos $N_{L}^{0}$ and $N_{R}^{0}$ :

$$
\left(\begin{array}{l}
v_{e L}^{0} \\
e_{L}^{-}
\end{array}\right), \quad\left(\begin{array}{c}
v_{\mu L}^{0} \\
\mu_{L}^{-}
\end{array}\right), \quad N_{L}^{0}, \quad N_{R}^{0}
$$

The neutrino mass term is

$$
-\mathcal{L}_{M}=m_{1}^{0} \bar{v}_{e L}^{0} N_{R}^{0}+m_{2}^{0} \bar{v}_{\mu L}^{0} N_{R}^{0}+M \bar{N}_{L}^{0} N_{R}^{0}+\text { H.c , }
$$

where $M$ is a large mass generated by a Higgs singlet or bare mass, and $m_{1,2}^{0}$ are small masses generated by the usual Higgs-doublet mechanism. Diagonalizing the mass matrix associated with $\mathcal{L}_{M}$ one finds that because of the mismatch between the left- and right-handed states there are two massless Weyl neutrinos $v_{1 L}$ and $v_{2 L}$ and one massive Dirac neutrino $v_{3}=v_{3 L}+v_{3 R}$, with mass $m_{3}=\left[\left(m_{1}^{0}\right)^{2}+\left(m_{2}^{0}\right)^{2}+M^{2}\right]^{1 / 2}$. These are related to the weak eigenstates by $v_{3 R}=N_{R}^{0}$ and by ${ }^{10}$ 


$$
\begin{aligned}
& v_{e L}^{0} \simeq v_{1 L}-\epsilon_{1} \epsilon_{2} v_{2 L}+\epsilon_{1} v_{3 L}, \\
& v_{\mu L}^{0} \simeq v_{2 L}+\epsilon_{2} v_{3 L}, \\
& N_{L}^{0} \simeq-\epsilon_{1} v_{1 L}-\epsilon_{2} v_{2 L}+v_{3 L},
\end{aligned}
$$

where

$$
\epsilon_{1}=\frac{m_{1}^{0}}{M}, \quad \epsilon_{2}=\frac{m_{2}^{0}}{M} .
$$

The states $v_{e L}^{0}$ and $v_{\mu L}^{0}$ are orthogonal $\left(\left\langle\nu_{e L}^{0} \mid v_{\mu L}^{0}\right\rangle=0\right)$. However, these are not the states that are actually produced in the low-energy weak processes. For $m_{3} \gg m_{K}$, for example, the states produced in the $K_{l 2}$ decays $K^{+} \rightarrow e^{+} v_{e L}$ and $K^{+} \rightarrow \mu^{+} \nu_{\mu L}$ are

$$
\begin{aligned}
& \left|v_{e L}\right\rangle=\frac{P_{l}\left|v_{e L}^{0}\right\rangle}{c_{L}^{v_{e}}} \simeq\left|v_{1 L}\right\rangle-\epsilon_{1} \epsilon_{2}\left|v_{2 L}\right\rangle, \\
& \left|v_{\mu L}\right\rangle=\frac{P_{l}\left|v_{\mu L}^{0}\right\rangle}{c_{L}^{v_{\mu}}} \simeq\left|v_{2 L}\right\rangle,
\end{aligned}
$$

where $P_{l}$ is the projection operator onto the subspace of light neutrinos, and $c_{L}^{v_{i}}$ are normalization factors. Clearly, $\left\langle v_{\mu L} \mid v_{e L}\right\rangle=-\epsilon_{1} \epsilon_{2} \neq 0$, so that lepton flavor is not conserved. For example, one could have the decay $K^{+} \rightarrow e^{+} v_{e L}$, followed by $v_{e L} n \rightarrow \mu^{-} p$, with the amplitude for the second reaction suppressed by $-\epsilon_{1} \epsilon_{2} \neq 0$; the net lepton-flavor violation for these reactions is $\Delta L_{e}=-1, \Delta L_{\mu}=+1$. In this example the total lepton number $L_{e}+L_{\mu}$ is conserved, but in more complicated models one could have $L$ violation as well. One could also have models in which the light neutrino masses are very small but nonzero.

\section{FORMALISM}

In this section we develop the formalism needed to describe nonorthogonal neutrinos in a very general class of models. Other aspects of quark and lepton mixing with heavy exotic particles are described in Ref. 5. We denote left- ( $\left.L_{-}\right)$and right- ( $R-$-) handed neutrinos by $n_{L}$ and $n_{R}^{c}$, respectively. The two are not independent but are related by $n_{R}^{c}=C \bar{n}_{L}^{T}$, where $C$ is the charge-conjugation matrix, i.e., $n_{L}$ and $n_{R}^{c}$ are essentially $C P$ conjugates. We usually do not differentiate between left-handed neutrinos and antineutrinos in our notations: there is no distinction between them in the general Majorana case, and in the special case of Dirac neutrinos both are represented by $n_{L}$ (Ref. 11).

If there are no exotic electric charges then there are only three possible $S U(2) \times U(1)$ assignments of the weak-eigenstate $L$ neutrinos

$$
\left[\begin{array}{l}
n_{O L}^{0} \\
e_{L}^{0-}
\end{array}\right], \quad\left[\begin{array}{l}
e_{L}^{0^{+}} \\
n_{E L}^{0}
\end{array}\right], \quad n_{S L}^{0},
$$

where $e_{L}^{0^{-}}$and $e_{L}^{0^{+}}$are weak-eigenstate charged leptons (e.g., $\left.e_{L}^{-}, \mu_{L}^{-}, \tau_{L}^{-}, \ldots\right)$ or antileptons (e.g., $\left.e_{L}^{+}, \mu_{L}^{+}, \tau_{L}^{+}, \ldots\right)$, respectively. The $n_{O L}^{0}$ are the "ordinary" SU(2) doublets, such as those in Eq. (1), those in new sequential families, or the left-handed parts of new vector doublets. 5,12 The $n_{E L}^{0}$ are "exotic" neutrinos occurring in doublets with left-handed antileptons. These are related by $C P$ to right-handed doublets

$$
\left(\begin{array}{l}
n_{E R}^{0^{c}} \\
e_{R}^{0^{-}}
\end{array}\right)
$$

such as occur in vector doublets or mirror families. To an excellent approximation ${ }^{5}$ the right-handed leptons $e_{R}^{-}$, $\mu_{R}^{-}$, and $\tau_{R}^{-}$are $\mathrm{SU}(2)$ singlets. Therefore, any such $R$ doublets must mainly involve new heavy charged leptons, although they could contain small admixtures of the known leptons. Finally, the $n_{S L}^{0}$ are $\mathbf{S U}(2)$ singlets. In the presence of general Majorana mass terms, the $n_{O L}^{0}$, $n_{E L}^{0}$, and $n_{S L}^{0}$ can all mix with each other. For Dirac neutrinos ( $L$ conserved) there is no mixing between the $n_{O L}^{0}$ $(L=+1)$ and the $n_{E L}^{0}(L=-1)$, and the $n_{S L}^{0}$ divide into two sectors with $L=+1$ and -1 ; these can mix with the $n_{O L}^{0}$ and $n_{E L}^{0}$, respectively.

It is convenient to arrange all of the weak-eigenstate $L$ neutrinos into a column vector

$$
n_{L}^{0}=\left(\begin{array}{l}
n_{O L}^{0} \\
n_{E L}^{0} \\
n_{S L}^{0}
\end{array}\right), \quad n_{R}^{0^{c}}=\left(\begin{array}{c}
n_{O R}^{0^{c}} \\
n_{E R}^{0^{c}} \\
n_{S R}^{0^{c}}
\end{array}\right),
$$

where $n_{O L}^{0}, n_{E L}^{0}$, and $n_{S L}^{0}$ are themselves column vectors consisting of the individual neutrino doublets.

We are concerned with a new type of lepton-number violation that is not directly related to the masses of the ordinary neutrinos. We therefore assume that all of the mass-eigenstate neutrinos are either "massless" (i.e., with masses too small to be kinematically relevant or to lead to observable neutrino oscillations, neutrinoless doublebeta decay, etc.), or "heavy" (i.e., too heavy to be directly relevant to ordinary weak processes). One could easily generalize to the case that both nonorthogonal states and neutrino mass effects are important simultaneously.

Let

$$
n_{L}=\left(\begin{array}{l}
n_{l L} \\
n_{h L}
\end{array}\right), \quad n_{R}^{c}=\left(\begin{array}{l}
n_{l R}^{c} \\
n_{h R}^{c}
\end{array}\right),
$$

where $n_{l L}$ and $n_{h L}$ are column vectors consisting of the light- (i.e., "massless") and heavy-mass-eigenstate neutrinos, respectively. The weak- and mass-eigenstate neutrinos are related by a unitary transformation

$$
n_{L}^{0}=U_{L} n_{L}, \quad n_{R}^{0^{c}}=U_{R} n_{R}^{c},
$$

where $U_{L}=U_{R}^{*}$ (Ref. 5). $U_{L}$ can be written in block form:

$$
U_{L}=\left(\begin{array}{cc}
A_{L} & E_{L} \\
F_{L} & G_{L} \\
H_{L} & J_{L}
\end{array}\right),
$$

where the submatrices $A_{L}, F_{L}$, and $H_{L}$ describe the overlap of the massless $v$ 's with ordinary $\left(n_{O L}^{0}\right)$ doublets, exotic $\left(n_{E L}^{0}\right)$ doublets, and singlets $\left(n_{S L}^{0}\right)$, respectively. Simi- 
larly, $E_{L}, G_{L}$, and $J_{L}$ are the corresponding overlaps of the heavy neutrinos. The unitarity of $U_{L}$ implies

$A_{L}^{\dagger} A_{L}+F_{L}^{\dagger} F_{L}+H_{L}^{\dagger} H_{L}=I, \quad A_{L} A_{L}^{\dagger}+E_{L} E_{L}^{\dagger}=I$,

so that $A_{L}$ is not unitary.

In Ref. 5 it is shown that the part of the leptoniccharged current involving the light neutrinos and charged leptons is (dropping the subscript $l$ )

$$
\begin{aligned}
\frac{1}{2} J_{W}^{\mu^{\dagger}} & =\sum_{i a}\left[\bar{n}_{i L} \gamma^{\mu}\left(A_{L}^{\dagger}\right)_{i a} c_{L}^{e} e_{a L}+\bar{n}_{i R}^{c} \gamma^{\mu}\left(F_{R}^{\dagger}\right)_{i a} s_{R}^{e_{a}} e_{a R}\right] \\
& =\bar{n}_{L} \gamma^{\mu} A_{L}^{\dagger} c_{L}^{e} e_{L}+\bar{n}_{R}^{c} \gamma^{\mu} F_{R}^{\dagger} s_{R}^{e} e_{R} \\
& =-\bar{e}_{R}^{c} \gamma^{\mu} c_{L}^{e} A_{L}^{*} n_{R}^{c}-\bar{e}_{L}^{c} \gamma^{\mu} s_{R}^{e} F_{L} n_{L}
\end{aligned}
$$

where $F_{R}=F_{L}^{*}$. In (14),

$$
e_{L}=\left(\begin{array}{llll}
e_{1 L} & e_{2 L} & \ldots
\end{array}\right)^{T}=\left(\begin{array}{llll}
e_{L}^{-} & \mu_{L}^{-} & \tau_{L}^{-} & \ldots
\end{array}\right)^{T}
$$

is a column vector of the charged leptons, while

$$
e_{L}^{c}=\left(\begin{array}{llll}
e_{1 L}^{c} & e_{2 L}^{c} & \ldots
\end{array}\right)^{T}=\left(\begin{array}{llll}
e_{L}^{+} & \mu_{L}^{+} & \tau_{L}^{+} & \ldots
\end{array}\right)^{T} .
$$

Similar definitions hold for $e_{R}$ and $e_{R}^{c}$. Also,

$$
\begin{aligned}
& c_{L}^{e}=\operatorname{diag}\left(\begin{array}{ccc}
c_{L}^{e_{1}} & c_{L}^{e_{2}} & \ldots
\end{array}\right), \\
& s_{R}^{e}=\operatorname{diag}\left(\begin{array}{ccc}
s_{R}^{e_{1}} & s_{R}^{e_{2}} & \ldots
\end{array}\right),
\end{aligned}
$$

are diagonal matrices, where $c_{L}^{e_{a}} \equiv \cos \theta_{L}^{e_{a}}$ represents the possible mixing of $e_{a L}$ with a heavy $L$-singlet charged lepton, and $s_{R}^{e_{a}} \equiv \sin \theta_{R}^{e_{a}}$ is the possible admixture of the mostly singlet $e_{a R}$ into an $R$ doublet. ${ }^{13}$

The first term in $J_{W}^{\mu}$ represents the nonuniversal reduction in strength of the left-handed charged current due to the neutrino and electron mixings, while the second is a right-handed current (RHC) induced by the mixing of the light neutrinos and charged leptons into heavy righthanded doublets. For small light-heavy mixings we expect $c_{L}^{e_{a}}$ to be close to unity and $A_{L}$ (or some submatrix of $A_{L}$ ) to be close to the identity; similarly, the $s_{R}^{e_{a}}$ and each component of $F_{L}=F_{R}^{*}$ are expected to be small.

From (14), the effective light state $n_{a L}$ produced in a weak decay such as $K^{+} \rightarrow e_{a R}^{+} n_{a L}$ is

$$
\left|n_{a L}\right\rangle \equiv \frac{\sum_{i}\left(A_{L}^{\dagger}\right)_{i a}\left|n_{i L}\right\rangle}{c_{L}^{v_{a}}}
$$

where

$\left(c_{L}^{v_{a}}\right)^{2}=\cos ^{2} \theta_{L}^{v_{a}} \equiv\left(A_{L} A_{L}^{\dagger}\right)_{a a}=\sum_{i}\left|\left(A_{L}^{\dagger}\right)_{i a}\right|^{2}$.

$n_{a L}$ is a coherent superposition of states $n_{i L}$. Since the $n_{i L}$ are degenerate (massless), $n_{a L}$ does not change in time except for an irrelevant overall phase. (If one allows the $n_{i L}$ to have small masses, then the components of $n_{a L}$ evolve separately, leading to normal neutrino oscillations.) Also, the decay rate is suppressed by $\left(c_{L}^{e_{a}}\right)^{2}\left(c_{L}^{v_{a}}\right)^{2}$ by mixing effects. The states $n_{a L}$ and $n_{b L}$ with $a \neq b$ are nonorthogonal. One has

$$
\lambda_{b a}^{L} \equiv\left\langle n_{b L} \mid n_{a L}\right\rangle c_{L}^{v_{a}} c_{L}^{v_{b}}=\left(A_{L} A_{L}^{\dagger}\right)_{b a},
$$

where, in general, $\lambda_{b a}^{L} \neq 0$. From (13), $\lambda_{b a}^{L}=-\left(E_{L} E_{L}^{\dagger}\right)_{b a}$. Since the relevant components of $E$ are small (they describe the admixture of heavy neutrinos into the ordinary neutrino states), $\lambda_{b a}^{L}$ is of second order in light-heavy mixing.

The second term in $J_{W}^{\mu_{W}^{\dagger}}$, if present, allows decays into the "wrong" helicity neutrinos (e.g., $K^{+} \rightarrow e_{a L}^{+} n_{a R}^{c}$ ) with the highly suppressed rate (compared to normal weak) $\left(s_{R}^{e_{a}}\right)^{2}\left(s_{R}^{v_{a}}\right)^{2}$, where

$$
\left(s_{R}^{v_{a}}\right)^{2}=\sin ^{2} \theta_{R}^{v_{a}} \equiv\left(F_{R} F_{R}^{\dagger}\right)_{a a}=\sum_{i}\left|\left(F_{R}^{\dagger}\right)_{i a}\right|^{2} .
$$

The corresponding light-neutrino states

$$
\left|n_{a R}^{c}\right\rangle \equiv \frac{\sum_{i}\left(F_{R}^{\dagger}\right)_{i a}\left|n_{i R}^{c}\right\rangle}{s_{R}^{v_{a}}}
$$

are again nonorthogonal, in general, with

$$
\lambda_{b a}^{R} \equiv\left\langle n_{b R}^{c} \mid n_{a R}^{c}\right\rangle s_{R}^{v_{a}} s_{R}^{v_{b}}=\left(F_{R} F_{R}^{\dagger}\right)_{b a} .
$$

The $C P$ conjugate of $n_{a R}^{c}$, produced in $K^{-} \rightarrow e_{a R}^{-} \bar{n}_{a L}$, for example, is

$$
\left|\bar{n}_{a L}\right\rangle \equiv \frac{\sum_{i}\left(F_{L}^{\dagger}\right)_{i a}\left|n_{i R}^{c}\right\rangle}{s_{R}^{v_{a}}},
$$

where the overbar distinguishes this state from $n_{a L}$ in (16). In the special case that $L$ is conserved, $n_{a L}$ and $\bar{n}_{a L}$ would be leptons and antileptons, respectively.

From (16), (20), and (22) we see that nonzero values for $\lambda_{b a}^{L}$ or $\lambda_{b a}^{R}$ for $a \neq b$, which are of second order in lightheavy mixing, indicate lepton-flavor violation. Similarly, nonzero values for

$\beta_{b a}^{L} \equiv\left\langle n_{b L} \mid \bar{n}_{a L}\right\rangle c_{L}^{v_{b}} s_{R}^{v_{a}}=\left(A_{L} F_{L}^{\dagger}\right)_{b a}=\left(F_{L} A_{L}^{\dagger}\right)_{a b}^{*}$

imply total lepton-number violation: i.e., $\beta_{b a}^{L} \neq 0$, which is linear in light-heavy mixing for Majorana neutrinos, describes the overlap of "neutrino" and "antineutrino" states.

\section{PHENOMENOLOGICAL IMPLICATIONS}

\section{A. Neutrino "oscillation" experiments}

Consider a weak decay into an $e_{a}^{+}$and its associated neutrino, e.g., $K^{+} \rightarrow e_{a}^{+} n_{a}$ (the discussion can easily be modified to the decay $K^{-} \rightarrow e_{a}^{-} n_{a}^{c}$ ). The decay can produce either the normal helicity state $n_{a L}$ in (16) or the wrong helicity state $n_{a R}^{c}$ in (20), with rates (relative to normal weak) $\left(c_{L}^{e_{a}}\right)^{2}\left(c_{L}^{v_{a}}\right)^{2}$ and $\left(s_{R}^{e_{a}}\right)^{2}\left(s_{R}^{v_{a}}\right)^{2}$, respectively. These neutrinos can subsequently rescatter in a target via the charged current to produce either the "right" lepton $e_{a L, R}$ or the "wrong" lepton $e_{b L, R}$. The cross sections relative to $\sigma_{0}$, the normal weak cross section in the absence of mixing, are 


$$
\begin{aligned}
& \frac{1}{\sigma_{0}} \sigma\left(n_{a L} \rightarrow e_{a L}\right)=\left(c_{L}^{e_{a}}\right)^{2}\left(c_{L}^{v_{a}}\right)^{2}, \\
& \frac{1}{\sigma_{0}} \sigma\left(n_{a L} \rightarrow e_{b L}\right)=\frac{\left(c_{L}^{e_{b}}\right)^{2}}{\left(c_{L}^{v_{a}}\right)^{2}}\left|\lambda_{b a}^{L}\right|^{2} \simeq\left|\lambda_{b a}^{L}\right|^{2}, \\
& \frac{1}{\sigma_{0}} \sigma\left(n_{a R}^{c} \rightarrow e_{a R}\right)=r\left(s_{R}^{e_{a}}\right)^{2}\left(s_{R}^{v_{a}}\right)^{2}, \\
& \frac{1}{\sigma_{0}} \sigma\left(n_{a R}^{c} \rightarrow e_{b R}\right)=r \frac{\left(s_{R}^{e_{b}}\right)^{2}}{\left(s_{R}^{v_{a}}\right)^{2}}\left|\lambda_{b a}^{R}\right|^{2},
\end{aligned}
$$

where $r=\sigma_{0}\left(v_{a R}^{c} \rightarrow e_{a R}^{c}\right) / \sigma_{0}\left(v_{a L} \rightarrow e_{a L}\right)$ is the ratio of antineutrino to neutron cross sections in the absence of mixing (e.g., for deep-inelastic scattering, $r \sim \frac{1}{3}$ if one ignores the antiquark content of the nucleon). The $n_{a R}^{c}$ cross sections in (24) are of $O\left(s^{4}\right)$, where $s$ is a typical light-heavy mixing angle. Since the $n_{a R}^{c}$ production rate is also of $O\left(s^{4}\right)$ we will ignore the rescatterings into $e_{a, b R}$ (the scatterings into antileptons are discussed below). The implications of the (small) suppression of the rate for $n_{a L} \rightarrow e_{a L}$, and also for rescattering via the neutral current, are discussed in Ref. 5. Here we concentrate on the lepton-flavor-violating process $n_{a L} \rightarrow e_{b L}$.

The sequence $K^{+} \rightarrow e_{a R}^{+} n_{a L}$ followed by $n_{a L} N \rightarrow e_{b L}^{-} X$ would mimic the effect of $n_{a L} \rightarrow n_{b L}$ in neutrino oscillation appearance experiments, ${ }^{2}$ except that the apparent “oscillation" probability

$$
P\left(n_{a} \rightarrow n_{b}\right)=\left|\lambda_{b a}^{L}\right|^{2} \simeq\left|\left\langle n_{b L} \mid n_{a L}\right\rangle\right|^{2}
$$

is independent of the neutrino energy or distance traveled. That is, if the light-mass eigenstates $n_{i L}$ are really massless (or extremely light) then $n_{a L}$ is independent of time. One would not see any characteristic oscillation signatures, only an excess of $e_{b}^{-}$events above background.

The Fermilab E531 Collaboration ${ }^{14}$ has presented upper limits on direct $\nu_{\mu} \tau$ and $\nu_{e} \tau$ couplings (as well as $v_{\mu} \rightarrow v_{\tau}$ and $v_{e} \rightarrow v_{\tau}$ oscillations). These imply

$$
\left|\lambda_{\tau \mu}^{L}\right|^{2}<0.002, \quad\left|\lambda_{\tau e}^{L}\right|^{2}<0.073
$$

at $90 \%$ C.L. (confidence limit) (the limits are collected in Tables I and II). Several groups ${ }^{15-18}$ have obtained limits on $v_{\mu} \rightarrow v_{e}$ oscillations in appearance experiments. They have not presented their results in terms of direct (energy and distance independent) probabilities. However, these can be obtained from the conventional neutrino oscillation exclusion plots. To see this, consider the usual oscillation formula

$$
P_{\mathrm{osc}}\left(v_{a} \rightarrow v_{b} ; L, p\right)=\sin ^{2} 2 \theta \sin ^{2} \frac{\Delta m^{2} L}{4 p},
$$

where $\theta$ is the $v_{a}-v_{b}$ mixing angle, $\Delta m^{2}=m_{1}^{2}-m_{2}^{2}$ is the difference of the squares of the neutrino-mass eigenvalues, $p$ is the neutrino energy, and $L$ is the distance traveled. In the large $\left|\Delta m^{2}\right|$ limit the second factor is just $\frac{1}{2}$, independent of $L$ and $p$. Hence, we can interpret upper limits on $\frac{1}{2} \sin ^{2} \theta$ in the large- $\left|\Delta m^{2}\right|$ region as limits on the direct probability $P\left(v_{a} \rightarrow v_{b}\right)$ in (25). At present the most stringent limit for large $\left|\Delta m^{2}\right|$ is the BNL result (Ref. 15) $\sin ^{2} 2 \theta<3.4 \times 10^{-3}$ (90\% C.L.), implying $\left|\lambda_{e \mu}^{L}\right|^{2}<0.0017$. The CHARM (Ref. 16), Big European Bubble Chamber (BEBC) (Ref. 17); and Los Alamos National Laboratory (LANL) (Ref. 18) experiments yield $\left|\lambda_{e \mu}^{L}\right|^{2}<0.004,0.01$, and 0.01 , respectively.

The PS191 experiment at CERN has reported ${ }^{19}$ a $3 \sigma$ excess of $23 \pm 8 v_{e}$ events above expected backgrounds in a $v_{\mu}$ beam. This would correspond to $\left|\lambda_{e \mu}^{L}\right|^{2}$ $\simeq 0.013 \pm 0.005$. However, the signal is in conflict with the BNL and, to a lesser extent, the other experiments, when interpreted either in terms of nonorthogonal neutrinos or conventional oscillations.

Nonorthogonal neutrinos do not lead to significant effects in neutrino disappearance experiments. ${ }^{2}$ This is partly because no oscillations occur. Furthermore, the $n_{a L}$ flux is generally monitored using the measured $\sigma\left(n_{a L} \rightarrow e_{a L}\right)$ cross section. Hence, the small absolute reduction in reaction rate from the $\left(c_{L}^{e_{a}}\right)^{2}\left(c_{L}^{v_{a}}\right)^{2}$ factor drops out.

In addition to producing the wrong lepton flavor, as in (25), nonorthogonal neutrinos can also lead to $\Delta L= \pm 2$ processes. The neutrinos $n_{a L}$ and $n_{a R}^{c}$ produced in $K^{+} \rightarrow e_{a}^{+} n_{a}$ can, in addition to the reactions in (24), rescatter into $e_{b}^{+}$(with $b=a$ or $b \neq a$ ) with cross sections

$$
\begin{aligned}
& \frac{1}{\sigma_{0}} \sigma\left(n_{a L} \rightarrow e_{b L}^{c}\right)=\frac{\left(s_{R}^{e_{b}}\right)^{2}}{\left(c_{L}^{v_{a}}\right)^{2}}\left|\beta_{a b}^{L}\right|^{2}, \\
& \frac{1}{\sigma_{0}} \sigma\left(n_{a R}^{c} \rightarrow e_{b R}^{c}\right)=r \frac{\left(c_{L}^{e_{b}}\right)^{2}}{\left(s_{R}^{v_{a}}\right)^{2}}\left|\beta_{b a}^{L}\right|^{2},
\end{aligned}
$$

which are of $O\left(s^{4}\right)$ and $O\left(s^{0}\right)$, respectively, for Majorana neutrinos. Hence, the total probability for the $\Delta L=2$ sequence $K^{+} \rightarrow e_{a}^{+} n_{a}$, followed by $n_{a}$ scattering to produce

TABLE I. 90\%-C.L. limits on lepton-flavor-violating parameters and expectations for the quadratic and linear seesaw models. $x$ is defined as $20 \mathrm{GeV} / M$, where $M$ is the heavy-neutrino mass.

\begin{tabular}{llll}
\hline \multicolumn{1}{c}{ Source } & \multicolumn{1}{c}{$\left|\lambda_{e \mu}^{L}\right|$} & \multicolumn{1}{c}{$\left|\lambda_{e \tau}^{L}\right|$} & \multicolumn{1}{c}{$\left|\lambda_{\mu \tau}^{L}\right|$} \\
\hline$v$ appearance & 0.041 & 0.27 & 0.045 \\
Unitarity (single parameter) & 0.0083 & 0.054 & 0.015 \\
Unitarity (simultaneous fit) & 0.082 & 0.11 & 0.095 \\
$\mu \rightarrow e \gamma$ & $9.5 \times 10^{-4}$ & & \\
Quadratic seesaw & $2.5 \times 10^{-7} x^{2}$ & $2.5 \times 10^{-6} x^{2}$ & $2.5 \times 10^{-4} x^{2}$ \\
Linear seesaw & $5 \times 10^{-4} x$ & $1.6 \times 10^{-3} x$ & $0.016 x$ \\
\hline \hline
\end{tabular}


TABLE II. 90\%-C.L. limits on $\Delta L= \pm 2$ parameters, and expectations for the quadratic and linear seesaw models; $x=20 \mathrm{GeV} / M$, where $M$ is a heavy-neutrino mass.

\begin{tabular}{lccll}
\hline \hline Quantity & $\begin{array}{c}\text { Experimental } \\
\text { limit }\end{array}$ & $\begin{array}{c}\text { Quadratic } \\
\text { seesaw }\end{array}$ & $\begin{array}{c}\text { Linear } \\
\text { seesaw }\end{array}$ & Source \\
\hline$\left|s_{R}^{e} \beta_{e e}^{L}\right|$ & $4 \times 10^{-7}$ & $2.5 \times 10^{-9} x^{2}$ & $4 \times 10^{-5} x$ & $\beta \beta_{0 v}$ \\
$\left|s_{R}^{\mu} \beta_{\mu \mu}^{L}\right|$ & 0.19 & $2.5 \times 10^{-5} x^{2}$ & $5 \times 10^{-3} x$ & $\bar{v}_{\mu} e \nrightarrow \mu^{-} v$ \\
$\left|s_{R}^{e} \beta_{\mu e}^{L}\right|$ & 0.045 & $2.5 \times 10^{-7} x^{2}$ & $5 \times 10^{-4} x$ & $\mu$ decay \\
& 0.041 & & & $v_{\mu} \rightarrow e^{+}$ \\
$\left|s_{R}^{\mu} \beta_{e \mu}^{L}\right|$ & 0.048 & $2.5 \times 10^{-5} x^{2}$ & $5 \times 10^{-3} x$ & $\mu$ decay \\
& 0.071 & & & $v_{\mu} \nrightarrow e^{+}$ \\
$\left|s_{R}^{\tau} \beta_{\mu \tau}^{L}\right|$ & 0.54 & $2.5 \times 10^{-3} x^{2}$ & $0.05 x$ & $\tau$ decay \\
$\left|s_{R}^{\tau} \beta_{e \tau}^{L}\right|$ & 0.43 & $2.5 \times 10^{-3} x^{2}$ & $0.05 x$ & $\tau$ decay \\
\hline \hline
\end{tabular}

$e_{b}^{+}$is

$$
P\left(n_{a} \rightarrow \bar{n}_{b}\right) \simeq\left(s_{R}^{e_{b}}\right)^{2}\left|\beta_{a b}^{L}\right|^{2}+r\left(s_{R}^{e}\right)^{2}\left|\beta_{b a}^{L}\right|^{2}
$$

relative to the normal $K^{+} \rightarrow e_{a}^{+} n_{a}, n_{a} \rightarrow e_{a}^{-}$, where the two terms are associated with intermediate $n_{a L}$ and $n_{a R}^{c}$, respectively. Similarly, the sequence $K^{-} \rightarrow e_{a}^{-} n_{a}^{c}$, $n_{a}^{c} \rightarrow e_{b}^{-}$, has the probability (relative to $n_{a}^{c} \rightarrow e_{a}^{+}$)

$$
P\left(\bar{n}_{a} \rightarrow n_{b}\right) \simeq\left(s_{R}^{e_{b}}\right)^{2}\left|\beta_{a b}^{L}\right|^{2}+\frac{1}{r}\left(s_{R}^{e_{a}}\right)^{2}\left|\beta_{b a}^{L}\right|^{2}
$$

The CHARM Collaboration ${ }^{20}$ has set an upper limit of 0.05 (90\% C.L.) on the effective cross section for $\bar{v}_{\mu} e^{-} \rightarrow \mu^{-} v$ relative to $\sigma\left(\nu_{\mu} e^{-} \rightarrow \mu^{-} v_{e}\right)$. This corresponds to $r P\left(\bar{v}_{\mu} \rightarrow v_{\mu}\right)=(1+r)\left(s_{R}^{\mu}\right)^{2}\left|\beta_{\mu \mu}^{L}\right|^{2}$ in our language (neglecting a negligible contribution from $\left.\left|\lambda_{e \mu}^{L}\right|^{2}\right)$. Hence, $s_{R}^{\mu}\left|\beta_{\mu \mu}^{L}\right|<0.19$. Similarly, one obtains $\left|s_{R}^{e} \beta_{\mu e}^{L}\right|<0.041$ and $\left|s_{R}^{\mu} \beta_{e \mu}^{L}\right|<0.071$ from the nonobservation of $e^{+}$in the BNL electron appearance experiment, ${ }^{15}$ which is equally sensitive to $e^{-}$and $e^{+}$. (The E531 $\tau$ appearance experiment ${ }^{14}$ is only sensitive to $\tau^{-}$.)

There are stringent experimental limits on

$$
R\left(l_{a}^{-} \rightarrow l_{b}^{-}\right)=\sigma\left(l_{a}^{-} N \rightarrow l_{b}^{-} N^{\prime}\right) / \sigma\left(l_{a}^{-} N \rightarrow v N^{\prime}\right)
$$

and on

$$
R\left(\mu^{-} \rightarrow e^{+}\right)=\sigma\left(\mu^{-} N \rightarrow e^{+} N^{\prime}\right) / \sigma\left(\mu^{-} N \rightarrow v N^{\prime}\right),
$$

where $N$ and $N^{\prime}$ are nuclei, both of which can occur as second-order weak processes with (nonorthogonal) neutrinos in the intermediate state. For example, $R\left(\mu^{-} \rightarrow e^{-}\right)<4 \times 10^{-12}$ (Ref. 21), while $R\left(\mu^{-} \rightarrow e^{+}\right)$ $<3 \times 10^{-10}$ (Ref. 22) at $90 \%$ C.L. These do not, however, imply useful constraints on $\lambda_{\mu e}$ or $\beta_{\mu e}$. This is partly because the amplitudes are second-order weak. In addition, $R\left(\mu^{-} \rightarrow e^{-}\right)$could occur at the tree level via a flavor-changing neutral current if the $\bar{e}-\mu-Z$ vertex were nonzero, so there is no way to separately bound the higher-order nonorthogonal neutrino effect. Finally, nuclear suppressions are enormous for $R\left(\mu^{-} \rightarrow e^{+}\right)$; one expects the rate to be many orders of magnitude below the experimental limit even for models with large- $L$ violation. ${ }^{23}$

\section{B. The solar-neutrino problem}

Nonorthogonal neutrinos do not by themselves yield a solution to the solar-neutrino problem. ${ }^{2}$

The propagation of massless neutrinos in matter can be described by introducing a time-dependent state vector

$$
|v(t)\rangle=\sum_{i=1}^{p} v_{i}(t)\left|n_{i L}\right\rangle,
$$

where the $n_{i L}$ are the $p$ light-mass eigenstates, and the coefficients $v_{i}(t)$ satisfy the Schrödinger-type equation ${ }^{24,2}$

$$
i \frac{d}{d t}\left(\begin{array}{c}
v_{1}(t) \\
\vdots \\
v_{p}(t)
\end{array}\right)=M\left(\begin{array}{c}
v_{1}(t) \\
\vdots \\
v_{p}(t)
\end{array}\right) .
$$

The matrix $M$ can be written as $M=M_{O}+M_{C}+M_{N}$. $M_{O}$, which is associated with the neutrino masses, vanishes for exactly massless neutrinos, while $M_{C}$ and $M_{N}$ represent the effect of coherent charged- and neutralcurrent forward scattering, respectively, of the neutrinos on matter.

From (14) the charged-current piece (from $n_{e} e^{-}$ $\left.\rightarrow e^{-} n_{e}\right)$ is

$$
M_{C}=\sqrt{2} G_{\mu} N_{e} A_{L}^{\dagger} P_{e} A_{L}
$$

where $G_{\mu}$ is the Fermi constant, $N_{e}$ is the electron density, and $P_{e}=\operatorname{diag}(100 \ldots 0)$ is the projection operator onto the $n_{e L}$. (In $M_{C}$ and $M_{N}$ we ignore unimportant electron and quark exotic mixing. ${ }^{5}$ )

The neutrino neutral current in the presence of mixing is 5

$$
\frac{1}{2} J_{Z}^{\mu}=\frac{1}{2} \bar{n}_{L} \gamma^{\mu}\left(A_{L}^{\dagger} A_{L}-F_{L}^{\dagger} F_{L}\right) n_{L},
$$

where the $A_{L}^{\dagger} A_{L}$ and $F_{L}^{\dagger} F_{L}$ terms are associated with the neutral currents of $n_{O L}^{0}$ and $n_{E L}^{0}$, respectively. One has ${ }^{2}$

$$
\begin{aligned}
M_{N} & =\sqrt{2} G_{\mu}\left(A_{L}^{\dagger} A_{L}-F_{L}^{\dagger} F_{L}\right)\left(g_{V}^{e} N_{e}+g{ }_{V}^{p} N_{P}+g_{V}^{n} N_{n}\right) \\
& \rightarrow-\frac{1}{2} \sqrt{2} G_{\mu} N_{n}\left(A_{L}^{\dagger} A_{L}-F_{L}^{\dagger} F_{L}\right),
\end{aligned}
$$

where $g_{V}^{i}$ and $N_{i}$ are the neutral-current vector coupling and density of particle $i$, respectively. The second form holds for a neutral medium $\left(N_{e}=N_{p}\right)$ when the standard-model values $g_{V}^{e}=-g p=-\frac{1}{2}+2 \sin ^{2} \theta_{W}, g_{V}^{n}$ 
$=-\frac{1}{2}$ are used.

First assume that all of the massless $L$ neutrinos correspond to the ordinary $L$ doublets $n_{O L}^{0}$, up to small lightheavy mixings. Then one can choose a basis such that $A_{L} \sim I$ up to small corrections, so that

$$
M=M_{C}+M_{N}=\sqrt{2} G_{\mu} N_{e}\left(P_{e}-\frac{x_{n}}{2} I\right)+M^{\prime}
$$

where the perturbation $M^{\prime}$ is a matrix associated with the small light-heavy mixing terms. In particular, $\boldsymbol{M}^{\prime}$ can have off-diagonal components from both $M_{C}$ and from $M_{N}$ (there is no reason for $A_{L}^{\dagger} A_{L}-F_{L}^{\dagger} F_{L}$ to be diagonal $^{25}$ ). In (36) $x_{n} \equiv N_{n} / N_{e}$. One expects $x_{n} \leq 0.17$ for the Sun, $x_{n} \sim 1.15$ in the iron core of a red giant, and $x_{n} \gg 1$ in a neutron star.

The propagation of an electron neutrino in the Sun can be obtained by solving (32) subject to the initial condition $|v(0)\rangle=\left|n_{e L}\right\rangle \sim\left|n_{1 L}\right\rangle$. The only major effect on the $n_{e L}$ flux occurs if there is a resonance, i.e., a location in which $M_{11}$ is degenerate with another diagonal component $M_{i i}$ of $M$. Then, for $M_{1 i}^{\prime} \neq 0$ the eigenvectors of $M$ include two which are $45^{\circ}$ mixtures of $n_{1 L}$ and $n_{i L}$, i.e., the effects of even tiny off-diagonal elements in $M^{\prime}$ are amplified. If the external densities vary sufficiently slowly with the distance from the center of the Sun, the initial $n_{e L}$ are adiabatically converted into $n_{i L}$. [Such a resonance would be exactly analogous to the MikheyevSmirnov-Wolfenstein ${ }^{24-27}$ (MSW) solution to the solarneutrino problem. In that case there is a degeneracy in the analogue of $M$ caused by a compensation between the charged-current coherent scattering term and the difference between two neutrino mass eigenvalues.]

Unfortunately, there is no resonance for $M$ in (36), i.e., $\left(P_{e}-x_{n} I / 2\right)_{11}=1-x_{n} / 2$ is never equal to any of the other diagonal components $\left(-x_{n} / 2\right)$ for any $x_{n}$.

If we relax the assumption that all of the massless states correspond to the $n_{O L}^{0}$ in the absence of mixing, then a resonance is possible, but only for specific large values of $x_{n}$. For example, if one of the $L$ singlets $n_{S L}^{0}$ corresponds approximately to a massless neutrino, then $A_{L}^{\dagger} A_{L}$ has a zero eigenvalue. The corresponding value of $M_{i i}$ would be degenerate with $M_{11}$ for $x_{n}=2$ and a resonance would occur if $n_{1 i} \neq 0$. However, the most natural models containing light $L$ singlets are those involving Dirac neutrinos. In that case the $L$ singlets are antineutrinos that do not mix with the neutrinos. Similarly, if there is an exotic doublet $n_{E L}^{0}$ with a massless neutrino, $F_{L}^{\dagger} F_{L}$ would have a unit eigenvalue, and a resonance could occur for $x_{n}=1$. Both models seem rather contrived, however, and in any case the resonances occur for much larger $x_{n}$ than is found in the Sun.

Therefore, nonorthogonal neutrinos do not by themselves offer a solution to the solar-neutrino problem. ${ }^{28}$ They do, however, allow an interesting variation on the usual MSW solution. It is well known that the MSW mechanism of matter-enhanced neutrino mixing can lead to the necessary (factor of 3 ) reduction in the event rate in the ${ }^{37} \mathrm{Cl}$ experiment ${ }^{27}$ for several bands of vacuum oscillation parameters, with $-\Delta m^{2}=m_{2}^{2}-m_{1}^{2}$ ranging from $10^{-8} \mathrm{eV}^{2}$ up to $5 \times 10^{-5} \mathrm{eV}^{2}$ and $\sin ^{2} 2 \theta$ down to $4 \times 10^{-4}$. If the conventional (i.e., without large nonorthogonal effects) MSW mechanism occurs for $v_{e} \rightarrow v_{\tau}$ conversion, then one needs $m_{v_{\tau}} \leq 10^{-2} \mathrm{eV}\left(m_{v_{a}}\right.$ is the mass of the dominant mass eigenstate of $\left.n_{a}^{0}\right)$. Assuming $m_{v_{e}}<m_{v_{\mu}}<m_{v_{\tau}}$ it would then be unlikely that any laboratory evidence for neutrino mass would ever be observed. For $v_{e} \rightarrow v_{\mu}$ in the Sun, it is conceivable that $m_{v_{\tau}}$ is in the $1-10 \mathrm{eV}$ range (and could possibly account for the dark matter ${ }^{2}$ ). In that case $\nu_{\mu} \leftrightarrow v_{\tau}$ oscillations would be the only observable effect.

However, it is possible that the MSW mechanism, with small masses in the $<10^{-2} \mathrm{eV}$ range, can coexist with nonorthogonal neutrinos. In that case there would still be the possibility of observing $L_{i}$ and $L$ violation (from the nonorthogonal neutrinos) in the laboratory.

$$
\text { C. } \mu \rightarrow e r
$$

Lepton-flavor violation associated with light-heavy mixing yields a nonzero amplitude for $\mu \rightarrow e \gamma$ (Ref. 29). Considering only the left-handed charged current for simplicity, there are two contributions associated with virtual light heavy-mass-eigenstate neutrinos, as shown in Fig. 1. The light-neutrino graph in Fig. 1(a) generates an amplitude proportional to $\lambda_{e \mu}^{L}$. However, this cancels against the mass-independent part of the heavy-neutrino graph in Fig. 1(b) because of the unitarity of $U_{L}$. The final result, therefore, depends on the heavy-neutrino masses. One finds (including the necessary Goldstone boson and self-energy diagrams)

$$
\begin{aligned}
& B(\mu \rightarrow e \gamma) \\
& \left.\qquad=\frac{3 \alpha}{2^{7} \pi}\left(c_{L}^{e}\right)^{2}\left(c_{L}^{\mu}\right)^{2}\left|2 \sum_{i}\left(E_{L}\right)_{e i} F\right| \frac{M_{i}^{2}}{M_{W}^{2}}\right)\left.\left(E_{L}^{\dagger}\right)_{i \mu}\right|^{2},
\end{aligned}
$$

where $M_{i}$ is the mass of the $n_{h i}, E_{L}$ is the light-heavy mixing matrix in (12), and $F(x)$ is the function

$$
F(x)=\frac{x\left(1-6 x+3 x^{2}+2 x^{3}-6 x^{2} \ln x\right)}{(1-x)^{4}},
$$

which varies slowly from 0 to 2 as $x$ ranges from 0 to $\infty$.

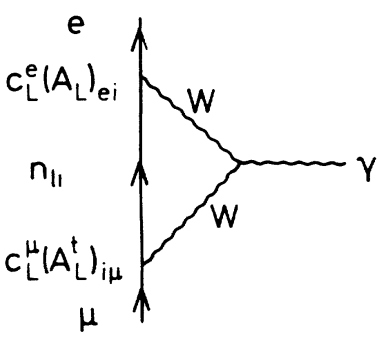

(a)

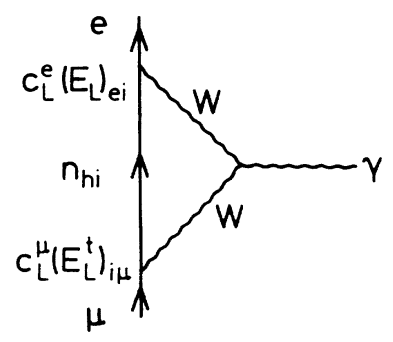

(b)
FIG. 1. (a) Light- and (b) heavy-neutrino contributions to $\mu \rightarrow e \gamma$. 
For $M_{i}=M_{W}$, for example, $F(x)=\frac{1}{2}$, so that, from (13) and (37),

$$
B(\mu \rightarrow e \gamma)=\frac{3 \alpha}{2^{7} \pi}\left(c_{L}^{e}\right)^{2}\left(c_{L}^{\mu}\right)^{2}\left|\left(A_{L} A_{L}^{\dagger}\right)_{e \mu}\right|^{2}
$$

We will use this as a typical estimate of $B(\mu \rightarrow e \gamma)$. However, the estimate is not rigorous because fine-tuned nondegenerate values of the $M_{i}$ could conceivably lead to cancellations and therefore, to smaller $B(\mu \rightarrow e \gamma)$. Using (39) and the experimental limit $B(\mu \rightarrow e \gamma)<4.9 \times 10^{-11}$ (90\% C.L.) (Ref. 30) one obtains $\left|\lambda_{e \mu}^{L}\right|<9.5 \times 10^{-4}$. The corresponding limits ${ }^{31}$ on $\tau \rightarrow e \gamma, \mu \gamma$ do not yield any useful constraints on $\left|\lambda_{b \tau}^{L}\right|$.

\section{Unitarity constraints}

One can derive constraints on the $\lambda_{a b}^{L}$ that do not directly involve $L_{i}$ or $L$ violation. From the unitary of $U_{L}$ one has

$$
\left|\lambda_{b a}^{L}\right| \leq\left[\left(s_{L}^{v_{a}}\right)^{2}\left(s_{L}^{v_{b}}\right)^{2}\right]^{1 / 2}
$$

[(40) follows by combining the Schwarz inequality for $\left(E_{L} E_{L}^{\dagger}\right)_{b a}$ with the unitarity relation (13)]. In Ref. 5 we have presented limits on the $\left(s_{L}^{v_{a}}\right)^{2}$ and the analogous charged-lepton and quark mixings, based on (a) the relation between $M_{W}, M_{Z}$, and the Fermi constant, (b) charged-current universality, (c) limits on induced RHC, and (d) flavor-diagonal neutral currents. It was found that there are enough constraints to limit all of the fermion mixings simultaneously. The corresponding limits on $\left|\lambda_{b a}^{L}\right|$ from (40) are presented in Table I for two cases: (a) only one mixing $\left(s_{L}^{v_{a}}\right)^{2}$ is allowed to be nonzero at a time, and (b) all of the lepton and quark mixings are allowed to be nonzero simultaneously, so that cancellations in the constraints are possible. ${ }^{32}$ It is seen that the latter constraints are much weaker (by an order of magnitude for $\left.\lambda_{e \mu}^{L}\right)$.

\section{E. Neutrinoless double-beta decay $\left(\beta \beta_{0 v}\right)$}

The light-neutrino-exchange diagram in Fig. 2, which involves both left- and right-handed leptonic currents

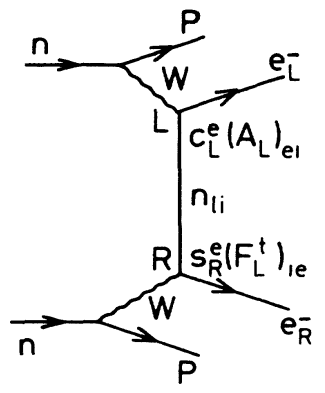

(a)

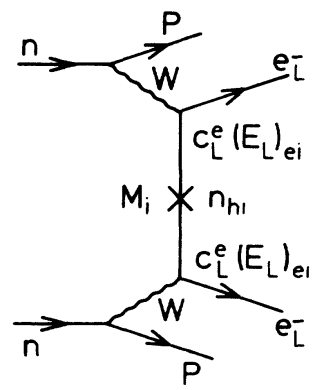

(b)
FIG. 2. (a) Induced RHC contribution to neutrinoless double-beta decay. (b) Heavy-neutrino Majorana mass contribution. yields a contribution to neutrinoless double-beta decay that survives even for massless neutrinos ${ }^{33}$ [an analogous phenomenon is well known for $\mathrm{SU}(2)_{L} \times \mathbf{S U}(2)_{R} \times \mathrm{U}(1)$ models]. Experimental limits on the relevant (leptonic RHC) parameter $\langle\eta\rangle$ imply $^{33}$

$$
|\langle\eta\rangle|=\left|c_{L}^{e} s_{R}^{e} \beta_{e e}^{L}\right|<0.4 \times 10^{-6} .
$$

The analogous LR diagram involving heavy neutrinos is smaller due to propagator effects. We mention for completeness that if the heavy neutrinos are Majorana there is a contribution to the effective Majorana mass ${ }^{33}\left\langle M_{v}\right\rangle$ for $\beta \beta_{0 v}$ from the heavy-neutrino-exchange diagram in Fig. 2(b). (This is a heavy particle effect that is not directly related to nonorthogonality.) One has

$$
\left\langle m_{v}\right\rangle=\left(c_{L}^{e}\right)^{2} \sum_{i}\left(E_{L e i}\right)^{2} \xi_{i} M_{i} F\left(A, M_{i}\right)
$$

where $E_{L}$ is the light-heavy mixing in (12), $M_{i}$ and $\xi_{i}= \pm 1$ are, respectively, the Majorana mass and $C P$ parity of $n_{h i}$, and $F\left(A, M_{i}\right)=\left\langle e^{-M_{i}^{r} r} / r\right\rangle /\langle 1 / r\rangle$ is a propagator factor which falls as $M_{i}^{-2}$ for $M_{i} \gg 1(\mathrm{MeV})$ (Ref. 23). Experimentally, $\left|\left\langle m_{v}\right\rangle\right| \leq 1 \mathrm{eV}$. The implications will be discussed in Sec. IV.

\section{F. Muon decay}

The induced RHC in (14) yield the effective muon decay couplings ${ }^{34}$

$$
\begin{aligned}
& \left|g^{L L}\right|^{2}=\left(c_{L}^{e}\right)^{2}\left(c_{L}^{\mu}\right)^{2}\left(c_{L}^{v_{e}}\right)^{2}\left(c_{L}^{v_{\mu}}\right)^{2}, \\
& \left|g^{R R}\right|^{2}=\left(s_{R}^{e}\right)^{2}\left(s_{R}^{\mu}\right)^{2}\left(s_{R}^{v_{e}}\right)^{2}\left(s_{R}^{v_{\mu}}\right)^{2}, \\
& \left|g^{L R}\right|^{2}=\left(c_{L}^{e}\right)^{2}\left(s_{R}^{\mu}\right)^{2}\left[\left(c_{L}^{v_{e}}\right)^{2}\left(s_{R}^{v_{\mu}}\right)^{2}+\left|\beta_{e \mu}^{L}\right|^{2}\right], \\
& \left|g^{R L}\right|^{2}=\left(s_{R}^{e}\right)^{2}\left(c_{L}^{\mu}\right)^{2}\left[\left(s_{R}^{v_{e}}\right)^{2}\left(c_{L}^{v_{\mu}}\right)^{2}+\left|\beta_{\mu e}^{L}\right|^{2}\right],
\end{aligned}
$$

where $g^{a b}$ is the strength of the decay amplitude for an $a$-handed electron and $b$-handed muon. The terms $\beta_{\mu e}^{L}$ and $\beta_{e \mu}^{L}$ represent the $\Delta L= \pm 2$ mixing between $n_{\mu L}$ and $\bar{n}_{e L}$ and between $n_{e L}$ and $\bar{n}_{\mu L}$, respectively. From the general analysis of $\mu$ decay constraints in Ref. 5, we obtain

$$
\left|s_{R}^{e} \beta_{\mu e}^{L}\right|<0.045, \quad\left|s_{R}^{\mu} \beta_{e \mu}^{L}\right|<0.048
$$

at $90 \%$ C.L. The spectrum parameter in leptonic $\tau$ decay also yields weak constraints on $\left|s_{R}^{\tau} \beta_{a \tau}^{L}\right|, a=\mu, e$ (Table II).

\section{DISCUSSION}

Mixing between ordinary neutrinos and the heavy neutrinos expected in many extensions of the standard model allows individual lepton-flavor and total lepton-number violation in the light sector, even if the light neutrinos are massless. ${ }^{6-9}$ That is because the projections of the orthogonal weak-eigenstate neutrinos onto the space of light neutrinos (which are the relevant states for most weak processes) are, in general, not orthogonal.

In this paper we have developed the formalism needed to describe such effects in a very general class of models. 
Lepton-flavor violation is described by the parameters $\lambda_{b a}^{L} \simeq\left\langle n_{b L} \mid n_{a L}\right\rangle$, which are of second order in lightheavy mixing. For Majorana neutrinos there is also the possibility of total lepton-number violations $(\Delta L= \pm 2)$. These are associated with the overlap between ordinary neutrinos, $n_{b L}$, and left-handed antineutrinos, $\bar{n}_{a L}$. The latter occur in doublets with charged antileptons

$$
\left(\begin{array}{l}
E_{L}^{+} \\
N_{L}
\end{array}\right)
$$

and are related by $C P$ to the right-handed doublets

$$
\left(\begin{array}{l}
N_{R}^{c} \\
E_{R}^{\bar{R}}
\end{array}\right)
$$

found in mirror models, $\mathrm{E}_{6}$ multiplets, etc. The relevant parameter is $s_{R}^{e_{a}} \beta_{b a}^{L} \simeq s_{R}^{e_{a}} s_{R}^{v_{a}}\left\langle n_{b L} \mid \bar{n}_{a L}\right\rangle$, where $s_{R}^{e_{a}}=\sin \theta_{R}^{e_{a}}$ and $s_{R}^{v_{a}}=\sin \theta_{R}^{v_{a}}$ describe the amount of admixture of the light leptons $e_{a R}$ and $\bar{n}_{a R}^{c}$ in the right-handed doublet.

We have described the phenomenological limits on the parameters $\lambda_{b a}^{L}$ from neutrino "oscillation" appearance experiments (nothing actually oscillates if the light neutrinos are massless), $\mu \nrightarrow e \gamma$, and indirect constraints from lepton-flavor-conserving charged- and neutral-current processes $^{5}$ (which are related by unitarity). The results, shown in Table I, are quite stringent for $\lambda_{e \mu}^{L}$, but are weak for $\lambda_{e \tau}^{L}$ and $\lambda_{\mu \tau}^{L}$. Similarly, limits on $\Delta L= \pm 2$ amplitudes from neutrinoless double-beta decay $\left(\beta \beta_{0 v}\right)$ and $\bar{v}_{\mu} e \nrightarrow \mu^{-} v$, as well as constraints on $n_{e L} \bar{n}_{\mu L}$ and $n_{\mu L} \bar{n}_{e L}$ interference in muon decay are presented in Table II. The most stringent limit is on $\left|s_{R}^{e} \beta_{e e}^{L}\right|$ from $\beta \beta_{0 v}$.

It is interesting to compare these limits with the theoretical expectations. Typically one expects [cf. (6)]

$$
\lambda_{b a}^{L} \sim \frac{m_{a}^{0} m_{b}^{0}}{M^{2}}, \quad s_{R}^{e_{a}} \beta_{b a}^{L} \sim\left(\frac{m_{a}^{0}}{M}\right)^{2},
$$

where $m_{a}^{0}$ is the typical light-heavy mixing term in the mass matrix for the ath family and $M$ is the typical heavy-lepton mass. The values of the $m_{a}^{0}$ are very model dependent, but a broad class of models will fall between the following two cases. (a) The quadratic seesaw. In this case the quark and charged-lepton masses $m_{a}$ are generated by the normal Higgs mechanism, with $m_{a}^{0}=O\left(m_{a}\right)$. (b) The linear seesaw. This is the situation in which the direct quark and charged-lepton masses are zero for some reason. They then acquire masses $m_{a}$ of $O\left(m_{a}^{0^{2}} / M\right)$ by light-heavy mixing. Hence, one expects

$$
\lambda_{a b}^{L} \sim\left(\frac{m_{a} m_{b}}{M^{2}}\right)^{\sigma}, \quad s_{R}^{e_{a}} \beta_{b a}^{L} \sim\left(\frac{m_{a}^{2}}{M^{2}}\right)^{\sigma},
$$

where $\sigma=1$ or $\frac{1}{2}$ for the quadratic and linear seesaws, respectively. We choose $m_{a}=1 \mathrm{MeV}, 100 \mathrm{MeV}$, and 1 $\mathrm{GeV}$ for the typical quark and charged-lepton masses of the three known families. ${ }^{35}$ The predictions of these two simple models for $M \sim 20 \mathrm{GeV}$ are listed in Tables I and II. It is seen that the experimental limits are much weaker than the expectations of the quadratic seesaw. The linear seesaw predictions are more optimistic. In some cases, the existing limits are comparable to expectations, and for $s_{R}^{e} \beta_{e e}^{L}$ the prediction is much larger than is allowed by $\beta \beta_{0 v}$.

Similarly, the heavy-neutrino-exchange diagram in Fig. 2(b) yields an effective $\beta \beta_{0 v}$ mass $\left|\left\langle m_{v}\right\rangle\right|$ $\sim\left(10^{-3-}-10^{-2}\right)$ eV $(20 \mathrm{GeV} / M)^{3}$ for the quadratic seesaw and $\sim(10-100) \mathrm{eV}(20 \mathrm{GeV} / M)^{2}$ for the linear seesaw, ${ }^{23}$ if the heavy neutrino is Majorana, to be compared with the experimental upper limit of $\sim 1 \mathrm{eV}$. Again, the linear seesaw suggests that this contribution to $\beta \beta_{0 v}$ may be observable for fairly small values of $M$.

It is possible for the nonorthogonal neutrino phenomena to coexist with small masses for the light neutrinos. In fact, any time that light masses of order $m_{n_{a}} \sim m_{a}^{0^{2}} / M$ are generated by a seesaw ${ }^{4}$ mixing with heavy neutrinos of mass $M$ one expects the light neutrinos to be nonorthogonal with $\lambda$ and $\beta$ given by (45). However, in this case the ordinary neutrino mass effects for neutrino oscillations and other $L_{i}$ - and $L$-violating processes will generally be much larger than the nonorthogonal effects. It is only when the light masses are much smaller than $m_{a}^{0^{2}} / M$ that both mechanisms can be important. An example of this was already given in the discussion of the solar-neutrino problem: it is possible that small $\left(\leq 10^{-2} \mathrm{eV}\right)$ masses may solve the solar $v$ problem via the ordinary MSW effect, while $L_{i}$ and $L$ violation observable in the laboratory could occur via nonorthogonal neutrinos.

We have tried to give a very general discussion of nonorthogonal neutrino effects without restricting ourselves to specific models or Higgs mechanisms. However, a few words on how the various scenarios could come about might be useful here. The simplest models are generalizations of the example in Eqs. (3) and (4). If the total lepton number is conserved (so that there is a clear distinction between neutrinos and antineutrinos) and if there is a mismatch between the number of $p$ of left-handed Weyl neutrinos and $q$ of right-handed Weyl neutrinos, then there will automatically be $|p-q|$ massless Weylstates which cannot find partners. These will generally be nonorthogonal, just as in the example, with $\lambda_{a b}$ given by (45). The light neutrinos could be given very small masses as a perturbation on this picture if the necessary $p-q$ right-handed partners (we take $p>q$ for definiteness) are added in a second stage, with very small Dirac masses $\ll m_{a}^{0^{2}} / M$.

Constructing a model for the Majorana case is more difficult (unless one simply resorts to fine-tuning to keep some of the masses small), but possible. One way is to mimic the mechanism invoked in the Dirac case, but with a nonstandard approximately conserved lepton number in the neutrino sector. Consider, for example, a model with one each of ordinary and exotic doublets and one neutrino singlet:

$$
\left(\begin{array}{c}
v^{0} \\
e^{0^{-}}
\end{array}\right)_{L}, \quad\left[\begin{array}{c}
E^{0^{+}} \\
N^{0}
\end{array}\right)_{L}, \quad N_{S R}^{0^{c}}, \quad e_{R}^{0^{-}}, \quad E_{L}^{0^{-}} .
$$

Suitable Higgs-boson representations and discrete symmetries can be found such that the allowed tree-level mass terms are 


$$
\begin{aligned}
& -\mathcal{L}_{v}=m_{v}^{0} \bar{v}_{L}^{0} N_{S R}^{0^{c}}+M_{v} \bar{N}_{L}^{0} N_{S R}^{0^{c}}+\text { H.c. }, \\
& -\mathcal{L}_{e}=m_{e}^{0} \bar{e}_{L}^{0} e_{R}^{0}+M_{e} \bar{E}_{L}^{0} E_{R}^{0}+\delta \bar{E}_{L}^{0} e_{R}^{0}+\text { H.c. , }
\end{aligned}
$$

where we assume that $m_{v}^{0} \ll M_{v},\left(m_{e}^{0}, \delta\right) \ll M_{e} . \mathcal{L}_{v}$ has a conserved nonstandard lepton number $L^{v}$, where $L_{v^{0}}^{v}=L_{N^{0}}^{v}=L_{N_{S R}^{0 c}}^{v}=1$. Hence, at the tree level there is one massless neutrino (mainly $v_{L}^{0}$ ) and one heavy "Dirac" neutrino (mainly $N_{L}^{0}+N_{S R}^{0^{c}}$ ). However, $N_{L}^{0}$ will have a small admixture (of order $m_{v}^{0} / M_{v}$ ) of massless neutrino, so that $\beta_{e e}^{L} \sim m_{v}^{0} / M_{v}$. In the charged-current sector the $\delta \bar{E}_{L} e_{R}$ term induces $E_{R}^{0}-e_{R}^{0}$ mixing, so that the

$$
\left[\begin{array}{l}
N_{R}^{0^{c}} \\
E_{R}^{0^{-}}
\end{array}\right]
$$

doublet contains a small admixture $s_{R}^{e} \sim \delta / M_{e}$ of the light-mass eigenstate $e_{R}^{-}$. The $\delta$ term also ensures that $E_{L}^{+}$(and therefore, $N_{L}^{0}$ ) is an antilepton, so that $L^{v}$ is not conserved by the theory as a whole. At the tree level, therefore, there is one massless neutrino, total (conventional) lepton-number violation, and $\beta_{e e}^{L}$ and $s_{R}^{e}$ both of order light-heavy mixing. Loop effects will generate a small radiative mass for the light neutrino since $L^{v}$ is violated by $\delta$. [It is essential that the form in (48) be enforced by a symmetry. Otherwise, the loop effects would be infinite and one would have to introduce extra counterterms into (48).] One could also introduce a small mass for the light neutrino perturbatively in a second stage (in addition to the radiative mass). This could be Majorana (induced by a Higgs-boson triplet $^{3}$ or by a seesaw ${ }^{4}$ with a second large mass scale $M^{\prime} \gg m_{v}$ ), or it could be a small Dirac mass.

Nonorthogonal neutrino effects will occur at some level in almost all models involving heavy neutrinos. In many cases they will be small compared to more conventional lepton-number-violating effects involving the lightneutrino masses. In other cases they could be important even when the mass effects are negligible. They provide an important reason to vigorously pursue searches for lepton-number violation, especially in processes such as $\mu \rightarrow e \gamma, v_{\mu} \leftrightarrow \nu_{\tau}$ "oscillations," and neutrinoless doublebeta decay.

\section{ACKNOWLEDGMENTS}

D.L. would like to thank P. Krawczyk, S. Uma Sankar, and H. Steger for helpful discussions. This work was supported by DESY, by the Alexander von HumboldtStiftung (P.L.), and by Department of Energy Grant No. DE-AC02-76-ERO-3071.
*Permanent address: Department of Physics, University of Pennsylvania, Philadelphia, PA 19104.

${ }^{1}$ G. 't Hooft, Phys. Rev. Lett. 37, 8 (1976); Phys. Rev. D 14, 3432 (1976); 18, 2199(E) (1978).

${ }^{2}$ For recent reviews of neutrino mass, see P. Langacker, in Neutrino Physics, edited by H. V. Klapdor (Springer, Berlin, in press). F. Boehm and P. Vogel, Physics of Massive Neutrinos (Cambridge University Press, Cambridge, England, 1987); J. D. Vergados, Phys. Rep. 133, 1 (1986); S. M. Bilenky and S. T. Petcov, Rev. Mod. Phys. 59, 671 (1987); R. Eichler, in Lepton and Photon Interactions, proceedings of the International Symposium on Lepton and Photon Interactions at High Energies, Hamburg, West Germany, 1987, edited by R. Rueckl and W. Bartel [Nucl. Phys. B Proc. Suppl. 3, 389 (1987)].

${ }^{3}$ G. B. Gelmini and M. Roncadelli, Phys. Lett. 99B, 411 (1981); H. Georgi et al., Nucl. Phys. B193, 297 (1983).

${ }^{4}$ M. Gell-Mann, P. Ramond, and R. Slansky, in Supergravity, edited by F. van Nieuwenhuizen and D. Freedman (NorthHolland, Amsterdam, 1979), p. 315; T. Yanagida, Prog. Theor. Phys. B135, 66 (1978).

${ }^{5}$ P. Langacker and D. London, preceding paper, Phys. Rev. D 38, 886 (1988).

${ }^{6}$ B. W. Lee et al., Phys. Rev. Lett. 38, 937 (1977); B. W. Lee and R. E. Shrock, Phys. Rev. D 16, 1444 (1977).

${ }^{7}$ D. Wyler and L. Wolfenstein, Nucl. Phys. B218, 205 (1983).

${ }^{8}$ S. L. Glashow, Phys. Lett. B 187, 367 (1987).

9J. Bernabeu et al., Phys. Lett. B 187, 303 (1987); J. W. F. Valle, ibid. 199, 432 (1987).

${ }^{10}$ In writing (5) we have kept the lowest-order nonzero term in each coefficient. Using the exact expressions would lead to the same conclusion.

${ }^{11}$ For example, a single Dirac neutrino has four components.
Two of these are (in conventional notation) the SU(2)-doublet states $v_{L} \leftrightarrow v_{R}^{c}$, which are normally referred to as the lefthanded neutrino, and right-handed antineutrino, respectively. In addition there are $\mathrm{SU}(2)$ singlets $N_{R} \leftrightarrow N_{L}^{c}$, which are the right-handed neutrino and left-handed antineutrino. In our notation we would denote $\left(v_{L}, N_{L}^{c}\right) \rightarrow\left(n_{1 L}, n_{2 L}\right)$ and $\left(\nu_{R}^{c}, N_{R}\right) \rightarrow\left(n_{1 R}^{c}, n_{2 R}^{c}\right)$.

${ }^{12}$ Vector doublets are pairs

$$
\left(\begin{array}{l}
N_{L} \\
E_{L}^{-}
\end{array}\right) \quad\left(\begin{array}{l}
N_{R}^{c} \\
E_{R}^{-}
\end{array}\right)
$$

of left- and right-handed doublets, such as occur in the 27 plet of $\mathbf{E}_{6}$.

${ }^{13}$ The fact that all charged-lepton mixings with exotic ( $L$ singlet, $R$-doublet) leptons can be parametrized by $c_{L}^{e}{ }^{a}$ and $s_{R}{ }^{e}{ }^{a}$ follows from the observed absence of flavor-changing neutral currents between the charged leptons.

${ }^{14}$ E531 Collaboration, N. Ushida et al., Phys. Rev. Lett. 57, 2897 (1986).

${ }^{15}$ BNL Collaboration, L. A. Ahrens et al., Phys. Rev. D 31, 2732 (1985).

${ }^{16}$ CHARM Collaboration, quoted by Eichler, in Lepton and Photon Interactions (Ref. 2).

${ }^{17}$ BEBC Collaboration, C. Angelini et al., Phys. Lett. B 179, 307 (1986).

${ }^{18}$ LANL Collaboration, T Bombeck et al., Phys. Lett. B 194, 591 (1987).

${ }^{19}$ PS191 Collaboration, G. Bernardi et al., Phys. Lett. B 181, 173 (1986).

${ }^{20}$ CHARM Collaboration, F. Bergsma et al., Phys. Lett. 122B, 465 (1983). 
${ }^{21}$ TRIUMF Collaboration, S. Ahmed et al., Phys. Rev. Lett. 59, 970 (1987).

${ }^{22}$ SIN Collaboration, R. Abela et al., Phys. Lett. 95B, 318 (1980).

${ }^{23}$ See, for example, J. D. Vergados, Phys. Rep. 133, 1 (1986).

${ }^{24}$ L. Wolfenstein, Phys. Rev. D 17, 2369 (1968); 20, 2634 (1979).

${ }^{25}$ Off-diagonal neutral currents were the original motivation for the study of matter effects on neutrino oscillations (Ref. 24).

${ }^{26}$ S. P. Mikheyev and A. Yu Smirnov, Yad. Fiz. 42, 1441 (1985) [Sov. J. Nucl. Phys. 42, 913 (1985)]; Nuovo Cimento 9C, 17 (1986).

${ }^{27}$ See Ref. 2 for a detailed discussion and complete set of references.

${ }^{28}$ The effects of mixings on the solar-neutrino problem have also been discussed by Valle (Ref. 9).

${ }^{29}$ S. T. Petcov, Yad. Fiz. 25, 641 (1977) [Sov. J. Nucl. Phys. 25, 340 (1977)]; W. Marciano and A. Sanda, Phys. Lett. 67B, 303
(1977); Lee et al. (Ref. 6); T. P. Cheng and L.-F. Li, Phys. Rev. Lett. 38, 381 (1977); 45, 1908 (1980).

${ }^{30}$ LAMPF Collaboration, R. D. Bolton et al., Phys. Rev. Lett. 56, 2461 (1986).

${ }^{31}$ H. J. Gerber, in Proceedings of HEP '87, International Europhysics Conference on High Energy Physics, Uppsala, Sweden, 1987, edited by O. Botner (Uppsala University, Uppsala, Sweden, 1987).

${ }^{32}$ We use the weakest $\left(\Lambda_{\mu}=2\right)$ constraints from the joint fits in Ref. 5.

${ }^{33}$ See, for example, Boehm and Vogel, Physics of Massive Neutrinos (Ref. 2), and M. Doi, T. Kotani, and E. Takasugi, Prog. Theor. Phys. Suppl. 83, 1 (1985).

${ }^{34} \mathrm{P}$. Langacker and $\mathrm{D}$. London (unpublished).

${ }^{35}$ We take $m_{1} \sim 1 \mathrm{MeV}$ as an appropriate value for leptonic processes. In Ref. 5 we chose $m_{1} \sim 10 \mathrm{MeV}$ because quarks were being considered as well. 